\title{
WHERE AND HOW TO MANAGE: OPTIMAL SELECTION OF CONSERVATION ACTIONS FOR MULTIPLE SPECIES
}

\author{
ASTRID J.A.VAN TEEFFELEN ${ }^{1,2} \&$ ATTE MOILANEN ${ }^{1}$ \\ ${ }^{1}$ Metapopulation Research Group, Department of Biological and Environmental Sciences, \\ University of Helsinki, P.O. Box 65 (Viikinkaari 1), FIN-00014 Helsinki, Finland; \\ ${ }^{2}$ Land Use Planning Group, Department of Environmental Sciences, Wageningen University, \\ P.O. Box 47, NL-6700 AA Wageningen, the Netherlands. \\ email: astrid.vanteeffelen@wur.nl
}

\begin{abstract}
Multiple alternative options are frequently available for the protection, maintenance or restoration of conservation areas. The choice of a particular management action can have large effects on the species occurring in the area, because different actions have different effects on different species. Together with the fact that conservation funds are limited and particular management actions are costly, it would be desirable to be able to identify where, and what kind of management should be applied to maximize conservation benefits. Currently available site-selection algorithms can identify the optimal set of sites for a reserve network. However, these algorithms have not been designed to answer what kind of action would be most beneficial at these sites when multiple alternative actions are available. We describe an algorithm capable of solving multi-species planning problems with multiple management options per site. The algorithm is based on benefit functions, which translate the effect of a management action on species representation levels into a value, in order to identify the most beneficial option. We test the performance of this algorithm with simulated data for different types of benefit functions and show that the algorithm's solutions are optimal, or very near globally optimal, partially depending on the type of benefit function used. The good performance of the proposed algorithm suggests that it could be profitably used for large multi-action multi-species conservation planning problems.
\end{abstract}

Key words. - benefit function, conservation planning, habitat restoration, optimisation, reserve selection, site selection algorithm.

Biodiversity conservation is not just a matter of reservation of natural habitats. Conservation encompasses a range of protection and management options that could be used to achieve conservation goals. Numerous examples exist where conservation planning involves decision making between these alternative options: When defining reserves, each reserve area can be assigned to one of several protection levels, such as strict nature reserve, national park or protected landscape (IUCN 1994). Once protected, sites oftentimes require management to maintain their quality, which can change for example through succession. Again, a number of alternative management options are available to maintain site quality. For example, to conserve semi-natural meadows overgrowth can be prevented by grazing with various herbivore species, or by mowing management, each of which can take place at various intensity levels (see for examples e.g. Köhler et al. 2005; Mitchley and Xofis 2005; Woodcock et al. 2005). Also in the field of habitat restoration decisions are required between multiple alternative management options: The planning of agri-environment schemes and biodiversity offsets-i.e. areas where habitat is restored in order to compensate for habitat loss elsewhere due to development activities - are examples where multiple management actions are available to improve habitat quality of degraded sites (Cuperus et al. 1999; Manchester et al. 1999; Ten Kate et al. 2004; Donald and Evans 2006).

Each of the management options available for conservation can have different effects on different species. For example, in the case of the grazing of a meadow, extensive grazing could be most beneficial to particular species, whereas other species benefit more from an intensive grazing regime (Pykälä 2003; Pöyry et al. 2004; Köhler et al. 2005). Since each site can only be managed in one particular way, careful planning of site management is important for reaching conservation aims. In addition, conservation budgets are typically limited and particular management actions can be costly, and hence only a fraction of sites can be protected or managed. Planning of conservation management 
actions therefore requires decisions concerning a) which sites will be chosen for conservation action and b) which actions will be applied to each of these sites.

The field of reserve design has a tradition in the use of optimization tools to select optimal reserve networks for a large number of species in a cost-effective manner (Margules and Pressey 2000; Cabeza and Moilanen 2001; Williams et al. 2004; Sarkar et al. 2006). These optimization methods account for a single value per species per site, which typically is information either about the density, probability of occurrence or presence-absence of the species at the site. The optimization methods, which are often called site-selection or reserve selection algorithms, select a set of sites based on species occurrences at sites and complementarity in order to achieve a conservation objective, such as maximizing species representation for a given budget. We point out however, that in many real-world conservation planning problems sites could potentially be managed in several different ways and that each of these management options can have a different effect on each species. This results in a different value for each species in each site under each management option. As a consequence, the site selection problem extends from selecting the optimal set of sites for a particular option, to selecting the optimal set of sites and the optimal management option for each site. One could think of this as an optimal conservation action allocation problem.

Currently available site selection algorithms were not designed to deal with multiple alternative options per site. Compared to the protect-or-not scenario, a multi-action site selection problem is computationally more complex for two reasons: First, the number of different solutions and consequently the search space size increases enormously. Second, complex trade-offs between species can occur, as the best action for one species could be suboptimal for another species (Van Teeffelen 2007). The data demands for a multi-action reserve selection problem are large as well, and these factors together may be the reason why little has been published concerning optimal multi-action conservation planning (but see Hof et al. (1994) and Bevers et al. (1995) for timing of forest harvest and Holzkämper et al (2006) for optimal land use planning).

In this paper we first illustrate with a small example the characteristics of a planning problem with multiple options per site and multiple species when using benefit functions to value species representation. Next, we present a formulation for the multi-species multi-action planning problem, in which the effect of an action can differ per species and per location, and costs can vary between actions and locations. The aim of optimization is to maximize the value of the chosen set of sites and actions, given that there is a cost constraint and that only one action can be chosen per site. We tested the algorithm with simulated data to demonstrate the optimality characteristics of the presented problem formulation and optimization algorithm. In addition to testing for the effects of different benefit functions on algorithm performance, we discuss the consequences of choosing a particular benefit function type for conservation, and give guidelines how our method can be used in realworld planning problems.

\section{METHODS}

Consider a situation in which multiple management actions could be applied to each site in a landscape, but only a single action can be executed per site. Due to budget limitations only a limited number of sites can be managed. In this case decision is required on which action to apply to which site, such that the benefit for all species is maximized for the given budget. To quantify the effect that each of the restoration actions would have on the species, we use the concept of a benefit function (Hof and Raphael 1993; Bevers et al. 1995; Arponen et al. 2005, 2007; Cabeza and Moilanen 2006). A benefit function $f_{j}\left[R_{j}(\mathbf{X})\right]$ is an increasing function of the representation of species $j R_{j}(\mathbf{X})$, which specifies how the conservation value of a network (set of sites $\mathbf{X}$ ) changes when the species' representation level changes (Figure 1; see Table 1 for a list of symbols used). Species representation can be understood as the expected abundance, number of occurrences, individuals or populations of a species in the set of selected sites, following chosen conservation actions. Such estimates can for example be obtained from predictive species distribution models (Guisan and Thuiller 2005; Elith et al. 2006). The actual value of species $j$ in the network, $V_{j}(\mathbf{X})$, is determined by the benefit function $f_{j}$ for the species and a species-specific weight $w_{j}$. This weight can be used to set different conservation priorities for different species; for example, one can set higher weights for endangered species (Arponen et al. 2005; Holzkämper et al. 2006; Moilanen 2007; Van Teeffelen et al. in review). The following 
equation gives the value of one species in the selected set of sites:

$V_{j}(\mathbf{X})=w_{j} f\left[R_{j}(\mathbf{X})\right]$

The total value of the network is then simply a sum over species-specific values:

$F(\mathbf{X})=\sum_{j} V_{j}(\mathbf{X})$

In contrast to target based planning, benefit functions account for how much the current level of species representation in the network is below

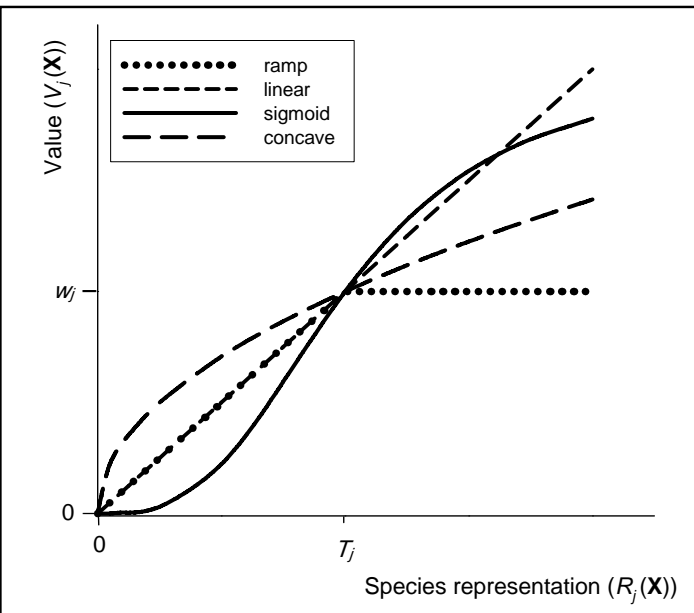

Figure 1. Graphical representation of the type of benefit functions used. The benefit functions translate the representation of species $j$ in the reserve network into a value, scaled by the weight given to the species $\left(w_{j}\right)$. At a chosen target level of representation $\left(T_{j}\right)$ the value will be equal to the species' weight $w_{j}$.

or above a given nominal target level of representation. The assumption is that increasing species representation is always better for species of conservation concern.

We next illustrate with a toy example how selection among multiple actions per site for multiple species can be handled with benefit functions. Assume a planning problem concerning a single site. This site could for example be a semi-natural meadow that requires management to avoid overgrowth. We assume two alternative management options to be available for this site: Option 1 concerns annual grazing of the site and option 2 concerns biennial grazing (every second year) of the site, both with cattle. Each of these options has an associated cost, which we assume to be 1.5 and 1 for options 1 and 2, respectively (Table 2). Assume that two species of conservation concern occur at the site. Species A has a population size of $a$ individuals, whereas the population size of species $\mathrm{B}$ is $b$ individuals, and we assume $a<b$. We assume that option 1 (annual grazing) is beneficial to species A, increasing its population size by $x$ individuals, while it does not influence species B. Likewise, we assume that option 2 (biennial grazing) is beneficial to species B, increasing its population size by $x$ individuals, while it does not affect species A. We used single-species effects for each option for the sake of simplicity; note however that each option could influence multiple species simultaneously, positively as well as negatively. Since only one of these management options can be applied to the site, a decision is required about which option is preferred.

The representation of each species is translated into a value by the benefit function, for the current representation levels, as well as for the expected representation levels under each of the conservation options. We demonstrate two different benefit functions: concave and sigmoid (Figure 2). Furthermore, we assume species to have equal conservation priority; hence we set weights for both species to 1. Although the species have an equal absolute increase in representation $(x)$ under their respective beneficial actions, the change in value that each of the options generates depends on the benefit function used. The concave benefit function returns a larger increase in value for option 1 than for option 2 (F1 > F2), and although this difference is partly counterbalanced by the higher cost of option 1, the difference in $\mathrm{F}$ relative to option cost (marginal gain, or efficiency) is larger for option 1 than for option 2 $\left(\Delta_{1}>\Delta_{2}\right.$, Table 2). Option 1 is therefore preferred using the concave benefit function type. The sigmoid function returns a larger increase in value for option 2 than for option 1 (F1 < F2), which is emphasised by the lower cost of option $2\left(\Delta_{1}<\Delta_{2}\right)$, and hence option 2 is preferred when using the sigmoid benefit function type. It follows that the optimal decision depends on which benefit function is used to value species representation. Note that this example corresponds to the cost-effective addition of one site to an existing set of sites.

The problem extends when the number of sites, options per sites and species increases. In 
addition, effects of actions on species as well as action cost can vary between sites, and species can be prioritized by incorporating different weights between them. Consequently, solving this kind of selection problem is no longer straightforward. We next describe an algorithm that is applicable to selection problems with multiple actions per site, and multiple species. The algorithm requires two main inputs: (i) a three-dimensional table giving the (estimated) representation of species $j$ at site $i$ given action $k$ and (ii) a table giving cost of action $k$ at site $i$. Our problem formulation, unlike target-based planning formulations, is mathematically

Table 1. Symbols used for multi-action conservation planning.

\begin{tabular}{ll}
\hline Symbol & Explanation \\
\hline $\mathbf{A}$ & set of available sites \\
$j$ & index for sites, $i=1,2, \ldots N_{s}$ \\
$k$ & index for species, $j=1,2, \ldots N_{p}$ \\
$r_{i j}(k)$ & index for different conservation actions, $k=1,2, \ldots N_{a}$ \\
$x_{i}$ & representation of species $j$ in site $i$ under action $k$ \\
$a_{i}$ & indicator variable, $x_{i}=1$ if site $i$ has been selected for action, else $x_{i}=0$ \\
$\mathbf{X}$ & action selected for site $i$ \\
& index set of sites that have been selected for a conservation action, i.e. the \\
$R_{j}(\mathbf{X})$ & network \\
$f_{j}\left[R_{j}(\mathbf{X})\right]$ & $=\sum_{i} x_{i} r_{i j}\left(a_{i}\right)$, representation of species $j$ in network $X$ given actions $a_{i}$ \\
& Benefit function: an increasing function of species representation $R_{j}(\mathbf{X})$, which \\
& expresses how the conservation value of $\mathbf{X}, F(\mathbf{X})$, changes when the \\
$w_{j}$ & representation level of species $j$ changes \\
& the weight of species $j$, to indicate a species' conservation priority relative to \\
$V_{j}(\mathbf{X})$ & other species under consideration \\
$F(\mathbf{X})$ & $=w_{j} f_{j}\left[R_{j}(\mathbf{X})\right]$, value of representation of species $j$ \\
$C_{i k}$ & $=\sum_{j} V_{j}(\mathbf{X})$, value of network $\mathbf{X}$ \\
$C_{\text {max }}$ & cost of action $k$ at site $i$ \\
$C_{u s e d}$ & the total budget available \\
$\Delta_{i k}$ & the part of the budget allocated \\
& $=[F(\mathbf{X}+i)-F(\mathbf{X})] / c_{i k}$, the marginal gain: the rate of increase of network value, \\
& relative to the cost of the site-action pair, when site $i$ is added to the network \\
& assuming action $k$ at site $i$
\end{tabular}

Table 2. Parameters and results for an example problem of multi-action selection. See Figure 2 for a graphical display of species representation and value.

\begin{tabular}{lccc}
\hline & Current & Option 1 & Option 2 \\
\hline Representation species A & $a$ & $a+x$ & $a$ \\
Representation species B & $b$ & $b$ & $b+x$ \\
Option cost & $c_{\text {current }}=0$ & $c_{1}=1.5$ & $c_{2}=1$ \\
Value & $F_{\text {current }}=V_{a}+V_{b}$ & $F_{1}=V_{a+x}+V_{b}$ & $F_{2}=V_{a}+V_{b+x}$ \\
Marginal gain & -- & $\Delta_{1}=\left(F_{1}-F_{\text {current }}\right) / c_{1}$ & $\Delta_{2}=\left(F_{2}-F_{\text {current }}\right) / c_{2}$ \\
Concave function, value & $F_{\text {current }}=1.50$ & $F_{1}=1.71$ & $F_{2}=1.62$ \\
marginal gain & & $\Delta_{1}=0.14$ & $\Delta_{2}=0.12$ \\
Sigmoid function, value & $F_{\text {current }}=1.03$ & $F_{1}=1.22$ & $F_{2}=1.35$ \\
marginal gain & & $\Delta_{1}=0.13$ & $\Delta_{2}=0.32$ \\
\hline
\end{tabular}



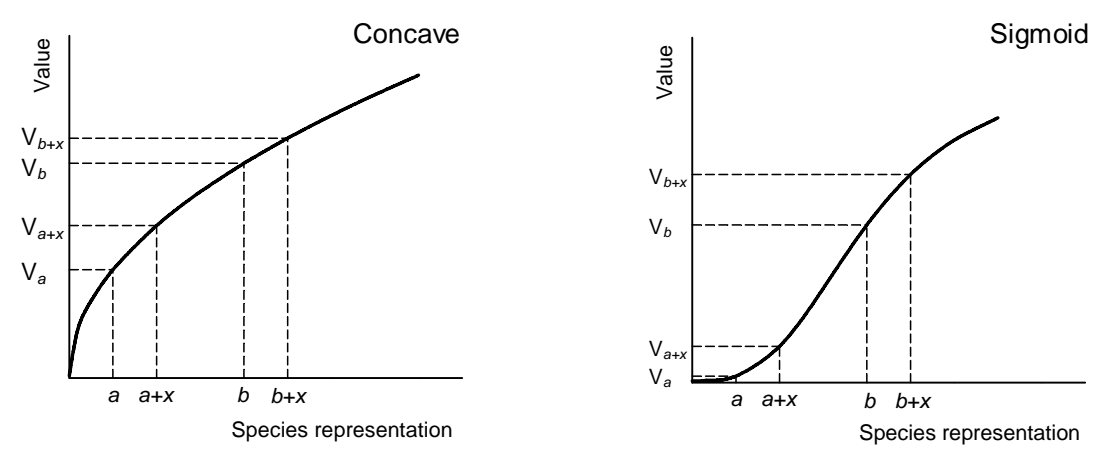

Figure 2. Translation of representation into value by a concave benefit function (left) and a sigmoid benefit function (right). $a$ = current representation of species A, $b=$ current representation of species $\mathrm{B}, x=$ increase in representation due to management action. $V$ represents a value corresponding to each representation level, according to the benefit function used. See Table 2 for an example.

concave, which suggests that a stepwise search strategy could be used to solve it in a globally optimal manner. Essentially, the proposed algorithm is a forward stepwise heuristic. It iteratively adds a site to the set of selected sites $(\mathbf{X})$, in combination with a particular management action for that site. The site-action pair that returns the highest marginal increase in conservation value $F$ to the set of selected sites, while accounting for action cost, is chosen to be added. After a site-action pair is added to the set of selected sites, no further actions are allowed for that site: the site is removed from the list of available sites. See Box 1 for a description of the algorithm, and Table 1 for explanation of symbols used. Stepwise heuristic algorithms are not in general guaranteed to find globally optimal solutions. The present formulation has however mathematical properties that suggest that an iterative heuristic might do very well in optimizing it. These properties include linear additivity over values for species, and a potentially linear or concave form for the benefit function for a species. We tested for the optimality of the algorithm's solutions by using simulated data sets, for which we could find the optimal solution by full enumerative search over all possible solutions. Full enumerative search is only possible for small problems due to computational limitations, whereas real-world problems may be large with many sites, species and options per site. Therefore heuristic algorithms are developed and used to provide (near-) optimal solutions for large problems. In order to verify whether certain problem characteristics affect the optimality, we defined a range of problem classes, each specified by a given number of sites, species, actions per site and available resource units (Table 3).

The value of the solution found by the algorithm, $F_{\text {alg }}(\mathbf{X})$, was compared to the value of the optimal solution $F_{\text {opt }}(\mathbf{X})$ that was found by full enumerative search over all possible solutions. The average value of all possible solutions, $F_{\text {mean }}(\mathbf{X})$, corresponds to the mean expected value when conservation actions are randomly assigned to locations, under the budget constraint. To check to what extent the algorithm's solutions are better than random allocation of conservation actions, we compared the difference between $F_{\text {opt }}(\mathbf{X})$ and $F_{a l g}(\mathbf{X})$ to the difference between $F_{\text {opt }}(\mathbf{X})$ and $F_{\text {mean }}(\mathbf{X})$ :

$Y_{a l g}=\frac{F_{\text {opt }}(\mathbf{X})-F_{\text {alg }}(\mathbf{X})}{F_{\text {opt }}(\mathbf{X})-F_{\text {mean }}(\mathbf{X})}$

where $Y_{a l g}$ is the relative performance of the algorithm. $Y_{a l g}<1$ indicates that the algorithm performs better than random, and $Y_{a l g}=0$ indicates that algorithm's solution is equal to the globally optimal solution. The performance of the algorithm might also depend on the type of benefit function, for which we tested four different types: a linear, ramp, concave and sigmoid function (Figure 1).

Each problem class was tested in combination with each benefit function in turn and 1000 replicates were created for each class-function pair by randomizing: 


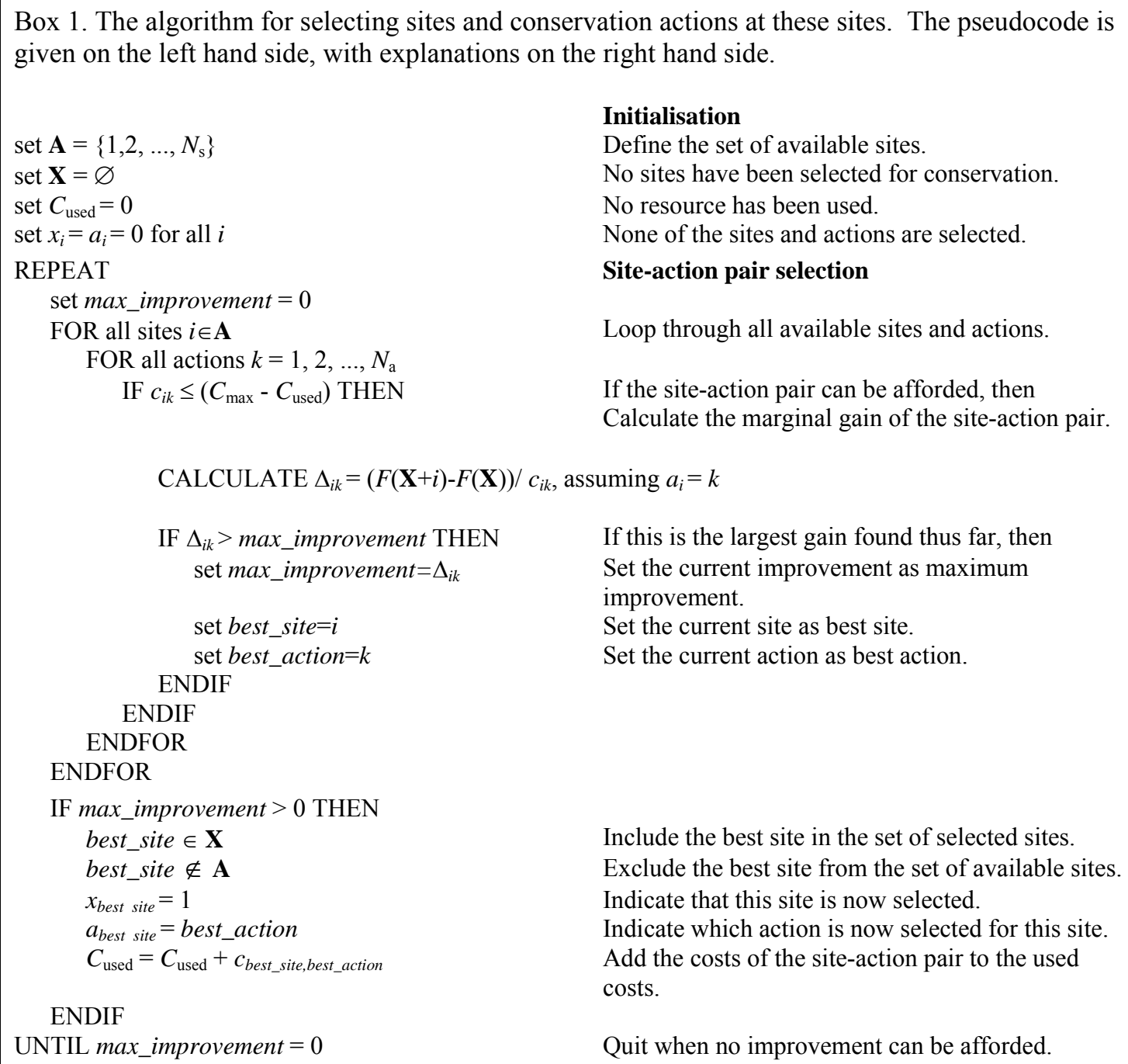

IF $\Delta_{i k}>$ max_improvement THEN set max_improvement $=\Delta_{i k}$

set best_site $=i$ set best_action $=k$

ENDIF

ENDIF

ENDFOR

ENDFOR

IF max_improvement $>0$ THEN

best_site $\in \mathbf{X}$

best_site $\notin \mathbf{A}$

$x_{\text {best site }}=1$

$a_{\text {best site }}=$ best_action

$C_{\text {used }}=C_{\text {used }}+c_{\text {best_site, best_action }}$

ENDIF

UNTIL max_improvement $=0$

If this is the largest gain found thus far, then Set the current improvement as maximum improvement.

Set the current site as best site.

Set the current action as best action.

Include the best site in the set of selected sites.

Exclude the best site from the set of available sites. Indicate that this site is now selected.

Indicate which action is now selected for this site. Add the costs of the site-action pair to the used costs.

Quit when no improvement can be afforded.

- $r_{i j}(k)$, the representation of each species $j$ in each site $i$ under management action $k$. Within each replicate, each site $i$ obtained a different random base-level of representation for each species $j$ (uniformly distributed between 0.0-1.0), on top of which each action $k$ added an extra contribution (uniformly distributed between 0.0-1.0).

- The weight of each species $w_{j} \in[1,5]$.

For simplicity, cost levels of various management actions $\left(c_{i k}\right)$ were kept equal at 1.0. The algorithm evaluates site-action pairs by their conservation value relative to cost (marginal gain), and since we varied conservation value already widely across sites, having equal cost levels per site-action pair should therefore not influence algorithm testing. The way the data was randomized, all of the problems had only a single unique globally optimal solution. We also tested the sensitivity of the algorithm to variation in problem characteristics. To do so, we varied the number of actions, the amount of resource available and the number of species of one problem class (D, Table 3), and created 1000 replicates of each modified problem. Only one factor was changed at a time, and solutions found by the algorithm were again compared to the globally optimal solution, relative to the mean of all solutions for that replicate. 
Table 3. Specifications of the problem classes tested.

\begin{tabular}{lrrrrr}
\hline Problem class specifications & A & B & C & D & E \\
\hline \# sites & 6 & 8 & 10 & 12 & 14 \\
\# species & 50 & 50 & 50 & 50 & 50 \\
\# actions per site & 3 & 3 & 3 & 3 & 3 \\
\# site-action pairs to select & 3 & 4 & 5 & 6 & 7 \\
Search space size* & 540 & 5670 & 61,236 & 673,596 & $7,505,784$ \\
\# randomized replicates & 1000 & 1000 & 1000 & 1000 & 1000 \\
$*=\left(\begin{array}{l}N \\
S\end{array}\right) S^{A}$, where $N=$ number of sites, $S=$ number of selected sites and $A=$ the number of possible actions per site.
\end{tabular}

\section{RESULTS}

We introduced an algorithm capable of optimizing conservation planning problems that have multiple actions per site. We applied the algorithm to simulated data and tested for the optimality of the algorithm's solutions. With linear and ramp type benefit functions, the algorithm always found the globally optimal solution. With concave and sigmoid benefit functions the proportion of successful globally optimal replicates varied depending on the problem class (Figure 3), where the concave function always returned a higher proportion of successful replicates than the sigmoid function. Overall, the success rates where remarkably high: for the concave function $>85 \%$ across all problem classes and for the sigmoid function $>50 \%$ (Figure 3a). Even though not all replicates returned a value equal to the value of the globally optimal solution, the algorithm's solutions were always close to optimal, proportional to the difference between the optimal solution and the mean value of all possible solutions. The average relative error for failed replicates across all tested problem classes was less than 0.08 from the global optimum for the sigmoid function and less than 0.04 for the concave function, compared to the mean value of all solutions (Figure 4a and e). Taken over all replicates, both failed and successful, these numbers are less than 0.005 and 0.02 for the concave and sigmoid benefit function respectively. Such errors are likely to be much smaller than observation and prediction errors for biodiversity distribution data. With increasing search space size (problem class $\mathrm{A} \rightarrow$ E, Table 3) the number of globally optimal replicates decreased for both the sigmoid and concave function (Figure 3a), but so did the average relative error (Figure $4 \mathrm{a}$ and e).

Algorithm sensitivity to problem characteristics (number of species, actions and resource units) was tested by comparing variants of problem class $\mathrm{D}$, varying one variable at a time. Ramp and linear benefit functions again always returned the globally optimal solutions, and the following results therefore concern the concave and sigmoid benefit functions only: With an increasing number of actions, the proportion of globally optimal replicates decreased, mainly for the sigmoid function (Figure 3b), with little or no effect on the relative error (Figure $4 b$ and $f$ ). The decrease in success rate is likely to be due to a major

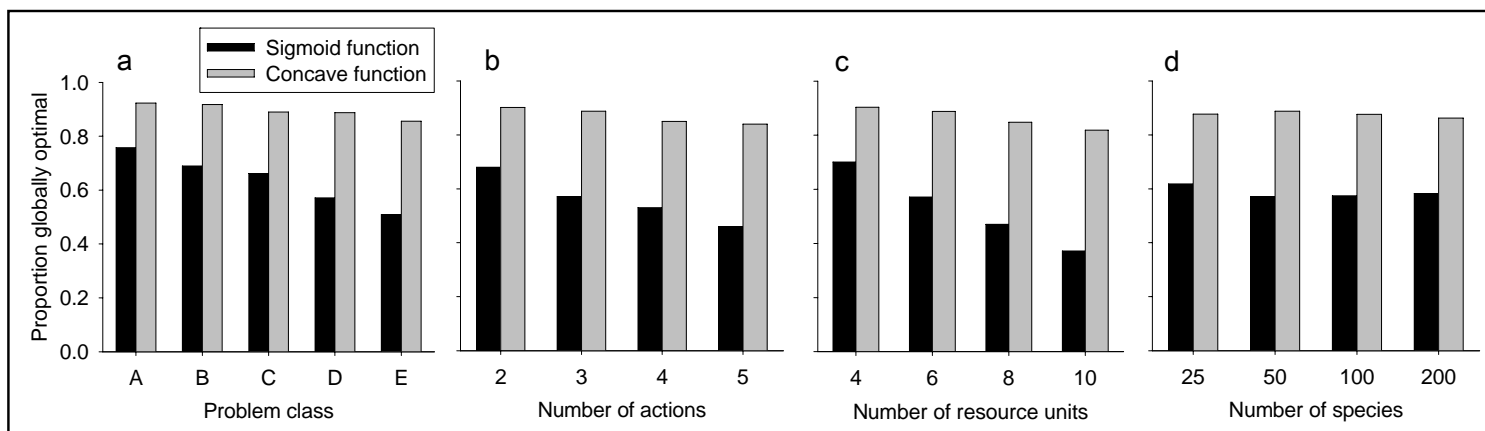

Figure 3. Proportion of replicates where the algorithm returned an objective function value equal to that of the globally optimal solution, for a concave and a sigmoid benefit function. Panel a displays success rates for problem definitions A-E (Table 3). Panels b-d display variants of problem $\mathrm{D}$, in which problem characteristics were varied one at a time. The characteristic modified is given on the $\mathrm{x}$-axis. 
increase in search space size. With an increasing resource level (more sites could be selected per replicate, because restoration cost was kept equal), the number of globally optimal replicates decreased (Figure 3c), again with little effect on the relative error of replicates where the algorithm failed to find the optimum (Figure 4c and g). Changing the number of species hardly influenced the proportion of optimal replicates (Figure 3d) and the relative error (Figure 4d and h).

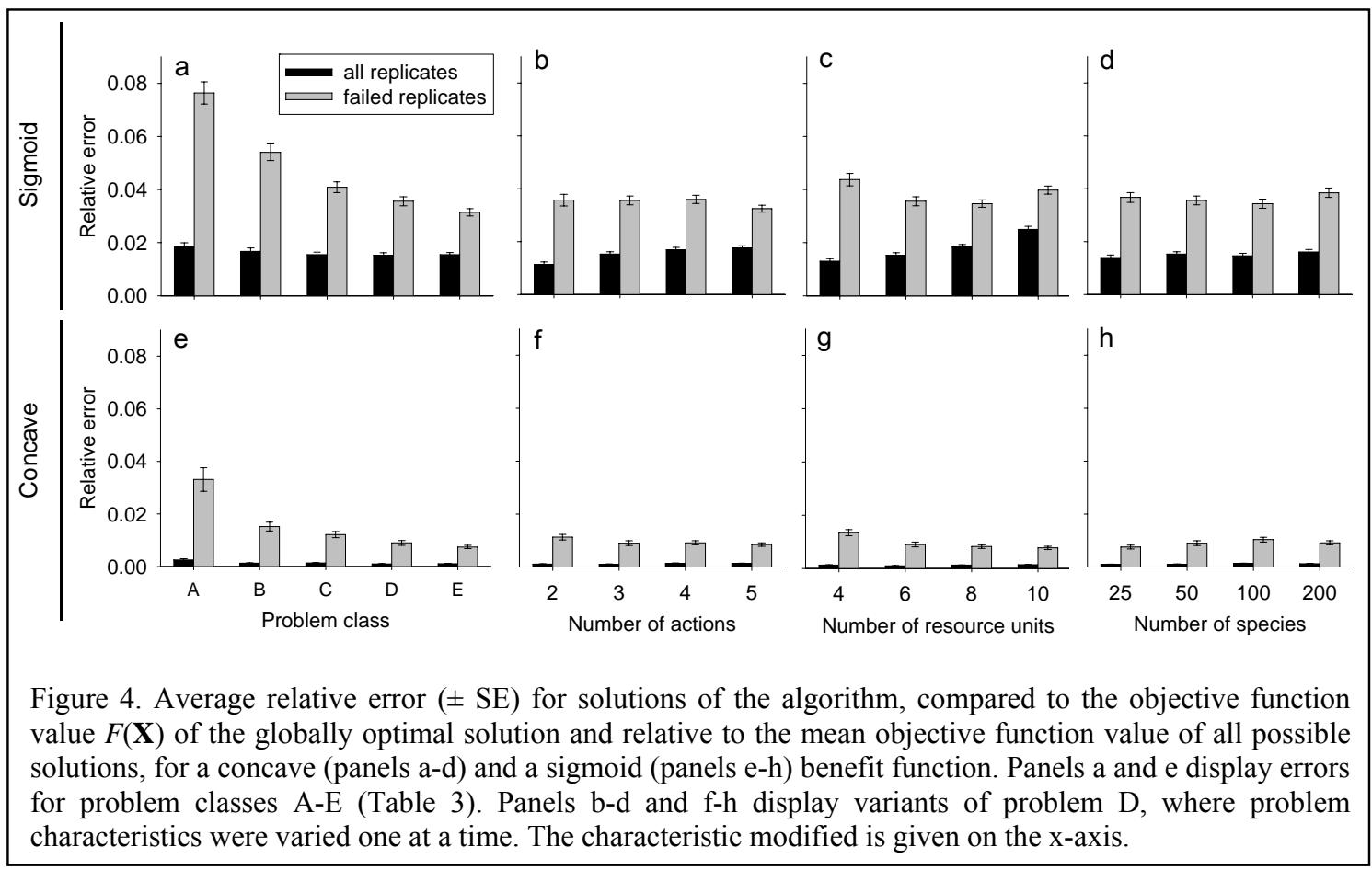

\section{DISCUSSION}

Although planning problems concerning multiple alternative management actions per site are common in practice, few approaches to solve these problems in an optimal manner have been published. The algorithm we introduced is capable of solving planning problems with multiple potential actions per site for both single and multiple species in an optimal, or nearoptimal manner. Potential applications of this type of decision support tool are for example found in the planning of mitigation and compensation measures (Race and Fonseca 1996; Cuperus et al. 2001); the targeting of management within protected area networks to maintain or enhance quality, or outside protected areas, in order to complement or buffer protected area networks through reserve selection; restoration (Hof et al. 2002; Pieterse et al. 2002; Cipollini et al. 2005) and agri-environment schemes (Kleijn et al. 2001; Donald and Evans
2006). Optimization tools such as the one presented in this paper can aid in more efficient and effective allocation of the available budgets for conservation (Pressey et al. 1997; Cowling et al. 2003). Earlier studies on multi-action planning (Hof et al. 1994; Bevers et al. 1995; Holzkämper et al. 2006) did not consider action cost in the optimization. Cost of actions can however differ widely, and conservation budgets are typically tight. The budget available is therefore expected to influence which set of sites and actions is optimal for a given set of species and given budget (Moilanen and Cabeza 2002).

The algorithm we used for multi-action planning is a stepwise heuristic, which have been criticized for returning sub-optimal results in conservation planning (Underhill 1994; Önal 2003). Optimization methods that guarantee global optimality, such as integer programming or stochastic dynamic programming, are generally favourable over methods that do not guarantee global optimality. However, global 
optimality comes with a price: both integer programming (see Williams et al. 2004) and stochastic dynamic programming (Westphal et al. 2003) have limitations to the sizes of data sets that can be analysed. The problem formulation presented here, has the interesting property that it can be solved optimally or almost optimally using a stepwise search strategy due to the mathematical characteristics of the benefit function. This allows for convenient formulation and solution of conservation planning problems, and results in relatively rapid computations for large conservation planning problems, which is advantageous for the use of conservation planning tools in interactive planning (Pressey et al. 1996; Sarkar et al. 2004).

The performance of the algorithm we propose is influenced by the type of benefit function employed to value species representations: namely whether the function is mathematically convex or concave, or not. The solutions for the ramp and linear benefit functions (which can be considered mathematically concave) were always globally optimal. Also, the success rates for the concave benefit function were very high and relative errors low, while results using a sigmoid (neither solely convex nor concave) benefit function were suboptimal. This supports our assumption that a stepwise search strategy should perform optimally or almost optimally on problems that are formulated with either convex or concave functions.

The mathematical properties of the concave benefit function allow the algorithm to find the global optimum with any given accuracy, if the landscape was divided into arbitrarily small land parcels. This follows because a concave or convex function can be optimised to an arbitrary precision using a gradient-ascent type optimisation method (Rockafellar 1970; Bazaraa et al. 1993). In our test with the concave benefit function formulation the global optimum was most often, though not always, found. Sometimes the global optimum was missed because the selection was done in discrete steps (by selecting sites-action pairs with discrete cost), which may cause slightly suboptimal behaviour due to the curvature of the benefit function. If the problem has more sites, then the optimal solution will consist of a larger number of selection units each of which make up a relatively smaller contribution of the total solution. As a consequence, the optimal solution should be relatively closer to the globally optimal one. This kind of behaviour is evident in
Figures $3 \mathrm{a}$ and $4 \mathrm{e}$, where the relative error of the stepwise search algorithm went down quickly as the problem sized increased. This demonstrates that the simple stepwise iterative algorithm can find practically optimal solutions for problems with many sites, when using problem formulation with a concave benefit function. The sigmoid function however, is neither convex nor concave. It therefore is relatively difficult to optimise, which is also demonstrated by suboptimal results in Figures 3 and 4 (average relative errors of up to $8 \%$ for failed replicates).

Optimality should however not be the key factor for choosing one or the other benefit function type to value species representation, but rather, biological reasons should determine the choice. We therefore next take a closer look at different benefit functions tested and outline how they affect the selection of conservation actions, to inform decision making with respect to valuing species representation. The use of linear and ramp benefit functions to value species representation always returned globally optimal solutions for our simulated data sets. Both functions value species representation linearly below a certain target level of representation. Above the target level, the ramp function is horizontal and does not value overrepresentation, whereas the linear function values over-representation equal to underrepresentation. A drawback of the linear formulation is the possibility of species to fully compensate each other: Increasing the representation of a better-represented species will generate the same increase in value as increasing the representation of a poorly represented species by an equal amount (see also Holzkämper et al. 2006). As a result, using linear functions to value species representations does not explicitly promote the selection of sites most beneficial to the least-represented species, which is a property we do not find appropriate for conservation planning.

The concave benefit function does value representation of species in a way that puts most emphasis on increasing the representation of the least-represented species, as we showed in Fig 2. In other words, there are decreasing marginal gains with increasing representation levels, which is a plausible way of translating representation to conservation value. Arponen et al. (2005) show that the use of a concave function benefits the representation of rare species, compared to using, for example, a step function. The concave formulation may be 
particularly suitable for plants or other species that are able to persist already in small areas (Arponen et al. 2005).

The sigmoid function is qualitatively very different. If there are not sufficient resources to cover all species at high representation levels, the sigmoid function has the tendency of obtaining high representation for some species while leaving others close to zero representation. Due to the function's S-shape, the total value of the network will increase more by adding a site that benefits a species with a representation level around the centre of the curve, than by selecting a site that benefits a species whose representation is at low levels close to the left part of the curve (Figure 2). When the aim is to maximize the representation of as many species as possible, this effect of the benefit function's shape may be undesirable. However, the sigmoid may be appropriate when there is reason to believe that the species can only persist at relatively high (meta)population sizes, in which case the sigmoid can be used to try to force the representation level of the species to an acceptably high level (Arponen et al. 2005). The step function, which is implied by target-based planning, essentially counts the number of species that have reached a target level of representation, without valuing under- and overrepresentation (Arponen et al. 2005). This step function is the extreme limit of the sigmoid function, in which the increase in representation levels of under-represented species is not valued at all, which may lead to solutions where some species achieve targets whereas the representation of other species remains close to zero.

How to use this method in practice? As mentioned in the introduction, the method requires estimated representations of each species at each site under each potential action, as well as costs of these actions. There are few examples where data are collected before and after a change in management, hence in most cases one needs to rely on estimates from e.g. species distribution models. These models can be used to link species occurrence to environmental variables such as climatic, soil and vegetation variables (Guisan and Thuiller 2005; Elith et al. 2006), but can also include conservation actions as predictor variables. For example, it could be considered to increase the amount of dead wood in particular forest patches as a management action, to benefit species depending on dead wood. Different actions under consideration could represent different amounts of dead wood. Species distribution models with dead wood as a predictor variable could next be used to obtain estimates of the effects of the different actions at each site. Van Teeffelen et al. (in review) provide a real-world example of the use of this method in the context of planning grassland management for a set of species with contrasting management requirements with respect to grazing intensity. If no quantitative data is available on the effects of different actions on a species, qualitative data such as expert opinion can also be used. Evidently there is uncertainty associated with such estimates, especially when management impacts are large, such as in the case of intensive restoration (Suding et al. 2004; Hilderbrand et al. 2005). Nevertheless, estimates from well calibrated and evaluated distribution models provide a sound scientific basis to justify allocating - oftentimes costly-management actions (Burgman et al. 2005). The method presented allows for a sensitivity analysis, such that the robustness of the results (selected sites and actions) to uncertainties in estimates of species responses and costs is analysed.

With respect to the parameterisation of a benefit function, it is possible to scale the function between a minimum and a maximum level of representation for each species. The minimum level of representation would be obtained by managing all sites in the least beneficial way for that species, and could be set as the null-representation in Figure 1. The maximum representation level is obtained by managing all sites in the most beneficial way for that species, and can be set equal to the target level of representation $T_{j}$ (Figure 1). All other solutions in terms of sites and actions selected, will then obtain values between 0 and $T_{j}$. The species-specific weight $w_{j}$ can be used to increase the slope of the benefit function, in order to prioritise particular species over other species. Again, a sensitivity analysis can help gaining insight in the effects of setting different priorities for different species (Van Teeffelen et al. in review).

Since habitats become increasingly fragmented due to human action, the spatial configuration of conservation networks is considered important for species persistence (Cabeza 2003; Opdam et al. 2003; Williams et al. 2005; Nicholson et al. 2006; Van Teeffelen et al. 2006). The algorithm we presented is non-spatial, but it could account for the spatial configuration of selected sites and actions implicitly by implementing for example a 
penalty term for boundary length or for distance to existing reserve networks or habitat remnants to induce qualitative clustering (see, e.g., Possingham et al. 2000; Cabeza et al. 2004; Crossman and Bryan 2006). Another way to implicitly account for connectivity is through the input data: as each action can have different effects on a species depending on the location of the action, one can adjust the estimated effect of a restoration action on a species, for example by connectivity to current populations of that species.

Multiple-action conservation problems where site value explicitly depends on the spatial configuration of selected sites and actions (Cabeza 2003; Moilanen 2005; Westphal et al. 2007), are categorically more complicated than any of the problem types analyzed in this paper. When multiple management actions are possible for each site, and the value of a site is influenced by the quality of neighbouring sites, the problem becomes spatially non-linear (the ultimate conservation value of a site depends on habitat quality and conservation actions taken at nearby sites). The spatial pattern of restoration actions influences occurrence levels of species in potentially conflicting ways: an action that is good for one species may be bad for another, and effects of connectivity influence the distribution of species. Finding the optimal set of sites and actions is therefore no longer straightforward. The problem may become even more complicated when species have diverse habitat requirements (for example different requirements in different stages of their life cycle). It is evident that further work is required to formulate general solutions for these more complicated, but nevertheless common types of multi-action conservation planning problems.

\section{ACKNOWLEDGEMENTS}

We thank M. Cabeza, A. Arponen, O. Ovaskainen and E. Meyke for helpful comments on an earlier version of the manuscript. This work was financially supported by the Academy of Finland, project \#202870.

\section{REFERENCES}

Arponen, A., R. K. Heikkinen, C. D. Thomas, and A. Moilanen. 2005. The value of biodiversity in reserve selection: Representation, species weighting, and benefit functions. Conservation Biology 19:2009-2014.

Arponen, A., H. Kondelin, and A. Moilanen. 2007. Area-based refinement for selection of reserve sites with the benefit-function approach. Conservation Biology 21:527-533.

Bazaraa, M. S., H. D. Sherali, and C. M. Shetty. 1993. Nonlinear Programming: Theory and Algorithms. John Wiley \& Sons, New York.

Bevers, M., J. Hof, B. Kent, and M. G. Raphael. 1995. Sustainable forest management for optimizing multispecies wildlife habitat: A coastal douglas-fir example. Natural Resource Modeling 9:1-23.

Burgman, M. A., D. B. Lindenmayer, and J. Elith. 2005. Managing landscapes for conservation under uncertainty. Ecology 86:2007-2017.

Cabeza, M. 2003. Habitat loss and connectivity of reserve networks in probability approaches to reserve design. Ecology Letters 6:665-672.

Cabeza, M., M. B. Araújo, R. J. Wilson, C. D. Thomas, M. J. R. Cowley, and A. Moilanen. 2004. Combining probabilities of occurrence with spatial reserve design. Journal of Applied Ecology 41:252-262.

Cabeza, M., and A. Moilanen. 2001. Design of reserve networks and the persistence of biodiversity. Trends in Ecology and Evolution 16:242-248.

Cabeza, M., and A. Moilanen. 2006. Replacement cost: A practical measure of site value for costeffective reserve planning. Biological Conservation 132:336-342.

Cipollini, K. A., A. L. Maruyama, and C. L. Zimmerman. 2005. Planning for restoration: A decision analysis approach to prioritization. Restoration Ecology 13:460-470.

Cowling, R. M., R. L. Pressey, R. Sims Castley, A. Le Roux, E. Baard, C. J. Burgers, and G. Palmer. 2003. The expert or the algorithm? - comparison of priority conservation areas in the Cape Floristic Region identified by park managers and reserve selection software. Biological Conservation 112:147-167.

Crossman, N. D., and B. A. Bryan. 2006. Systematic landscape restoration using integer programming. Biological Conservation 128:369-383.

Cuperus, R., M. Bakermans, H. A. U. De Haes, and K. J. Canters. 2001. Ecological compensation in Dutch highway planning. Environmental Management 27:75-89.

Cuperus, R., K. J. Canters, H. A. Udo de Haes, and D. S. Friedman. 1999. Guidelines for ecological compensation associated with highways. Biological Conservation 90:41-51.

Donald, P. F., and A. D. Evans. 2006. Habitat connectivity and matrix restoration: the wider implications of agri-environment schemes. Journal of Applied Ecology 43:209-218.

Elith, J., C. H. Graham, R. P. Anderson, M. Dudik, S. Ferrier, A. Guisan, R. J. Hijmans, F. Huettmann, J. R. Leathwick, A. Lehmann, J. Li, L. G. Lohmann, B. A. Loiselle, G. Manion, C. Moritz, M. Nakamura, Y. Nakazawa, J. M. Overton, A. T. Peterson, S. J. Phillips, K. Richardson, R. 
Scachetti-Pereira, R. E. Schapire, J. Soberon, S. Williams, M. S. Wisz, and N. E. Zimmermann. 2006. Novel methods improve prediction of species' distributions from occurrence data. Ecography 29:129-151.

Guisan, A., and W. Thuiller. 2005. Predicting species distribution: offering more than simple habitat models. Ecology Letters 8:993-1009.

Hilderbrand, R. H., A. C. Watts, and A. M. Randle. 2005. The myths of restoration ecology. Ecology and Society 10:19.

Hof, J., M. Bevers, L. Joyce, and B. Kent. 1994. An integer programming approach for spatially and temporally optimizing wildlife populations. Forest Science 40:177-191.

Hof, J., M. Bevers, D. W. Uresk, and G. L. Schenbeck. 2002. Optimizing habitat location for black-tailed prairie dogs in southwestern South Dakota. Ecological Modelling 147:11-21.

Hof, J., and M. G. Raphael. 1993. Some mathematical programming approaches for optimizing timber age-class distributions to meet multispecies wildlife population objectives. Canadian Journal of Forest Research 23:828-834.

Holzkämper, A., A. Lausch, and R. Seppelt. 2006. Optimizing landscape configuration to enhance habitat suitability for species with contrasting habitat requirements. Ecological Modelling 198:277-292.

IUCN. 1994. Guidelines for protected area management categories. CNPPA with the assistance of WCMC. IUCN, Gland, Switserland an Cambridge, UK.

Kleijn, D., F. Berendse, R. Smit, and N. Gilissen. 2001. Agri-environment schemes do not effectively protect biodiversity in Dutch agricultural landschapes. Nature 413:723-725.

Köhler, B., A. Gigon, P. J. Edwards, B. Krusi, R. Langenauer, A. Luscher, and P. Ryser. 2005. Changes in the species composition and conservation value of limestone grasslands in northern Switzerland after 22 years of contrasting managements. Perspectives in Plant Ecology, Evolution and Systematics 7:51-67.

Manchester, S. J., S. McNally, J. R. Treweek, T. H. Sparks, and J. O. Mountford. 1999. The cost and practicality of techniques for the reversion of arable land to lowland wet grassland - an experimental study and review. Journal of Environmental Management 55:91-109.

Margules, C. R., and R. L. Pressey. 2000. Systematic conservation planning. Nature 405:243-253.

Mitchley, J., and P. Xofis. 2005. Landscape structure and management regime as indicators of calcareous grassland habitat condition and species diversity. Journal of Nature Conservation 13:171183.

Moilanen, A. 2005. Reserve selection using nonlinear species distribution models. American Naturalist 165:695-706.
Moilanen, A. 2007. Landscape zonation, benefit functions and target-based planning: Unifying reserve selection strategies. Biological Conservation 134:571-579.

Moilanen, A., and M. Cabeza. 2002. Single-species dynamic site selection. Ecological Applications 12:913-926.

Nicholson, E., M. I. Westphal, K. Frank, W. A. Rochester, R. L. Pressey, D. B. Lindenmayer, and H. P. Possingham. 2006. A new method for conservation planning for the persistence of multiple species. Ecology Letters 9:1049-1060.

Önal, H. 2003. First-best, second-best, and heuristic solutions in conservation reserve site selection. Biological Conservation 115:55-62.

Opdam, P., J. Verboom, and R. Pouwels. 2003. Landscape cohesion: an index for the conservation potential of landscapes for biodiversity. Landscape Ecology 18:113-126.

Pieterse, N., A. Verkroost, M. Wassen, H. Olde Venterink, and C. Kwakernaak. 2002. A decision support system for restoration planning of stream valley ecosystems. Landscape Ecology 17:69-81.

Possingham, H. P., I. Ball, and S. Andelman. 2000. Mathematical methods for identifying representative reserve network. Pp. 291-306 in S. Ferson, and M. Burgman, eds. Quantitative methods for conservation biology. Springer, New York.

Pöyry, J., S. Lindgren, J. Salminen, and M. Kuussaari. 2004. Restoration of butterfly and moth communities in semi-natural grasslands by cattle grazing. Ecological Applications 14:1656-1670.

Pressey, R. L., H. P. Possingham, and J. R. Day. 1997. Effectiveness of alternative heuristic algorithms for identifying indicative minimum requirements for conservation reserves. Biological Conservation 80:207-219.

Pressey, R. L., H. P. Possingham, and C. R. Margules. 1996. Optimality in reserve selection algorithms: When does it matter and how much? Biological Conservation 76:259-267.

Pykälä, J. 2003. Effects of restoration with cattle grazing on plant species composition and richness of semi-natural grasslands. Biodiversity and Conservation 12:2211-2226.

Race, M. S., and M. S. Fonseca. 1996. Fixing compensatory mitigation: what will it take? Ecological Applications 6:94-101.

Rockafellar, R. T. 1970. Convex analysis. Princeton University Press, New Jersey.

Sarkar, S., C. Pappas, J. Garson, A. Aggarwal, and S. Cameron. 2004. Place prioritization for biodiversity conservation using probabilistic surrogate distribution data. Diversity and Distributions 10:125-133.

Sarkar, S., R. L. Pressey, D. P. Faith, C. R. Margules, T. Fuller, D. M. Stoms, A. Moffett, K. A. Wilson, K. J. Williams, P. H. Williams, and S. Andelman. 2006. Biodiversity conservation planning tools: 
Present status and challenges for the future. Annual Review of Environment and Resources 31:123-159.

Suding, K. N., K. L. Gross, and G. R. Houseman. 2004. Alternative states and positive feedbacks in restoration ecology. Trends in Ecology and Evolution 19:46-53.

Ten Kate, K., J. Bishop, and R. Bayon. 2004. Biodiversity offsets: Views, experience, and the business case. Pp. 95. IUCN, Gland, Switzerland and Cambridge, UK and Insight Investment, London, UK.

Underhill, L. G. 1994. Optimal and suboptimal reserve selection algorithms. Biological Conservation 70:85-87.

Van Teeffelen, A. J. A. 2007. Where and how to conserve: Extending the scope of spatial reserve network design. PhD-thesis. Department of Biological and Environmental Sciences. University of Helsinki, Finland.

Van Teeffelen, A. J. A., M. Cabeza, and A. Moilanen. 2006. Connectivity, probabilities and persistence: Comparing reserve selection strategies. Biodiversity and Conservation 15:899-919.

Van Teeffelen, A. J. A., M. Cabeza, J. Pöyry, K. M. Raatikainen, and M. Kuussaari. in review. Maximizing conservation benefit for grassland species with contrasting management requirements.

Westphal, M. I., S. A. Field, and H. Possingham. 2007. Optimizing landscape configuration: A case study of woodland birds in the Mount Lofty Ranges, South Australia. Landscape and Urban Planning 81:56-66.

Westphal, M. I., M. Pickett, W. M. Getz, and H. P. Possingham. 2003. The use of stochastic dynamic programming in optimal landscape reconstruction for metapopulations. Ecological Applications 13:543-555.

Williams, J., C. S. ReVelle, and S. A. Levin. 2004. Using mathematical optimization models to design nature reserves. Frontiers in Ecology and the Environment 2:98-105.

Williams, J. C., C. S. ReVelle, and S. A. Levin. 2005. Spatial attributes and reserve design models: A review. Environmental Modeling and Assessment 10:163-181.

Woodcock, B. A., R. F. Pywell, D. B. Roy, R. J. Rose, and D. Bell. 2005. Grazing management of calcareous grasslands and its implications for the conservation of beetle communities. Biological Conservation 125:193-202. 